MARS

\section{Mariners reach Mars}

THEse are busy weeks for steerable radio telescopes. Parkes (New South Wales) and Goldstone (California) have been tracking Apollo 11, Jodrell Bank has been concentrating on Luna 15 and, next week and the week after, Goldstone will be following the Mars probes which are reaching their destination after a journey of five months and something like 200 million miles.

Neither probe will be able to detect the presence of life, but the hope is that they will determine whether the martian environment is suitable for life to develop. And the question of martian canals should be settled once and for all by the television pictures which are to be radioed back.

Both Mariners were launched from Cape Kennedy, Mariner 6 on February 24 and Mariner 7 on March 27, and they are to make close approaches to Mars on July 30 (10.18 p.m. PDT) and August 4 (10.00 p.m. $\left.\mathrm{PD}^{\prime} \mathrm{T}\right)$ respectively. Each Mariner is to look at a different part of the planet-Mariner 6 is to photograph the equator and Mariner 7 part of the southern hemisphere and the polar cap. The two probes are the second stage in NASA's exploration of Mars. They follow Mariner 4 which in 1964-65 photographed Mars with a best resolution of two miles (compared with 100 miles for terrestrial telescopes). The next step is scheduled for $197 \mathrm{l}$, when it is planned to place two spacecraft in orbit for two months, followed by two soft landings-the Viking project-in 1973.

As well as the television cameras, next week's Mariners have a complement of instruments which makes them the most sophisticated planetary spacecraft so far. Radiometers will produce infrared maps to compare with the television pictures, and should enable people to decide whether the ice caps are water or solid carbon dioxide. The constituents of the upper atmosphere are to be analysed by an ultraviolet spectrometer, and those of the lower atmosphere and surface by an infrared spectrometer. Chemicals such as water, carbon dioxide, methane, ethylene and acetylene can be detected, in some cases in concentrations down to two parts per million. Atmospheric pressures and densities will be measured from the effect on the telemetry as the Mariners swing behind Mars, when the signals will have to pass through the atmosphere. As a bonus, radio tracking of the spacecraft orbits throughout their flights should improve the values of some astronomical parameters - the mass of Mars, the mass ratio of the Earth and the Moon and the distance from the Earth to Mars when the Mariners arrive.

If all goes well, the television pictures will have a best resolution of 900 feet, and colour filters which can swing in front of the lenses will allow colour pictures to be built up. Each Mariner will begin taking pictures a few days before the closest approach, and a complete coverage of the planet except for the pole caps should be achieved as the planet rotates. (A martian day is 24.6 hours.) Altogether, 143 approach pictures are to be taken if communications with the spacecraft are up to scratch. Otherwise, a more conservative sequence of eight approach pictures by each Mariner is planned. If all goes well, the first pictures should be appearing on the monitors at Goldstone at 18.35 (PDT) on July 29. One of the martian moons, Phobos, could appear on the approach sequence. Close-ups of the surface will begin to be taken 15 minutes before the time of closest approach, with one picture every 42 seconds. Every seventh picture element will be trans. mitted back to Earth in real time, and a complete picture will be replayed from tapes after the encounter. Each Mariner is programmed to take twelve high resolution and twelve medium resolution pictures during the close approach. understand. Professor Brown thought the indications were that the rocks are lava and that the fine dust is meteoritic.

Two points particularly attracted the attention of Professor S. Tolansky of the Royal Holloway College, University of London, who is to prospect in the samples for diamonds. One was the way the fine dust stuck to the astronauts' boots, which Professor Tolansky attributed to electrostatic charges on the dust due to bombardment by the solar wind. The second point has to do with Professor Tolansky's interest in tektites, and a remark which he heard from one of the astronauts that there was something glassy-looking. Professor Tolansky believes this was either a tektite, or a splash of glassy material generated by the heat of an impacting object.

The team led by Dr J. E. Geake of the Institute of Science and Technology at Manchester University, which will be investigating optical emissions and the optical polarization of specimens, is interested in the dlust layer. Mr G. Walker was gratified that the lunar surface was lighter than the material immediately beneath. This seems to have been caused by radiation damage of the topmost soil. One problem in the past has been that laboratory experiments with protons suggest that most particles darken under radiation, but it now looks as if vacuum pump oil was getting into the equipment and darkening the specimens. He added that the colour of the lunar surface seems to match that of the powdered basalt which the Manchester team has had in its equipment. Dr Geake commented that the behaviour of the dust bears out the results of experiments by Dr Salisbury at the Air Force Cambridge Laboratories. There it was found that if almost any dust is pumped down to $10^{-10}$ torr to represent lunar conditions, an adsorbed layer of air molecules which remains in softer vacuums is stripped off. Van der Waals forces then come into play between the grains and the dust behaves like a wet soil. In the Air Force Cambridge experiments, dust sifted through fine wires built up on the wires like wet snow.

Professor A. W. Bishop of Imperial College, London, who is not involved with the analysis of samples, commented on the Moonwalk from the point of view of soil mechanics, especially the way the lunar soil behaves as if it had a caked surface. Electrostatic forces giving apparent cohesion is one explanation. More likely is that the dust is extremely rough, something like pulverized coke. This is expected to be the case on the Moon where there is no weathering to produce the smooth round particles which are usually encountered on the Earth. The particles then lock together and transmit stresses better than terrestrial dusts. 